\title{
THE INTRINSIC RANDOM FUNCTIONS AND THEIR APPLICATIONS
}

\author{
G. MATHERON, Centre de Morphologie Mathématique, Fontainebleau
}

\begin{abstract}
The intrinsic random functions (IRF) are a particular case of the Guelfand generalized processes with stationary increments. They constitute a much wider class than the stationary RF, and are used in practical applications for representing non-stationary phenomena. The most important topics are: existence of a generalized covariance (GC) for which statistical inference is possible from a unique realization; theory of the best linear intrinsic estimator (BLIE) used for contouring and estimating problems; the turning bands method for simulating IRF; and the models with polynomial GC, for which statistical inference may be performed by automatic procedures.

GUELFAND GENERALIZED STOCHASTIC PROCESS; INTRINSIC RANDOM FUNCTION; GENERALIZED COVARIANCES; POLYNOMIAL COVARIANCE; TURNING BANDS METHOD
\end{abstract}

\section{TABLE OF CONTENTS}

0. INTRODUCTION $\ldots$.

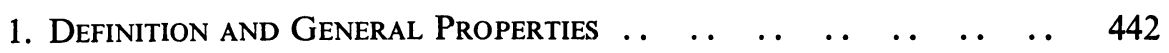

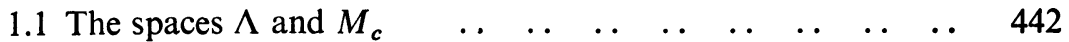

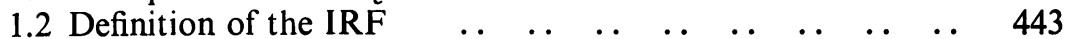

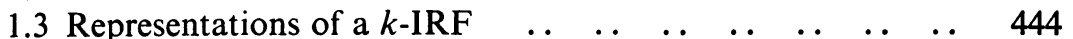

1.4 The translations formula $\ldots$.

1.5 A decomposition theorem ..

1.6 Drift of an IRF $\quad \ldots \quad$. .

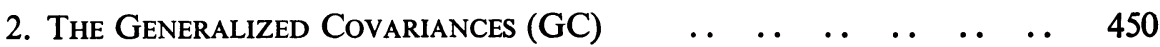

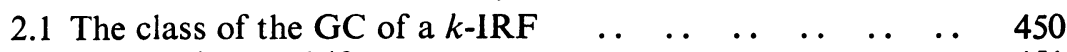

$\begin{array}{lllllllllll}2.2 & k \text {-IRF without drift } & \ldots & \ldots & \ldots & . & \ldots & . & \ldots & . & 451\end{array}$

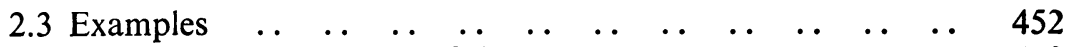

2.4 Applications of Theorem 2.1 .

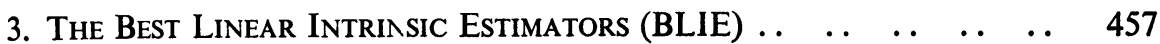

Example 3.1 (the finite case) $\ldots$.

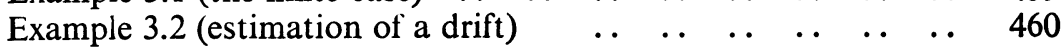

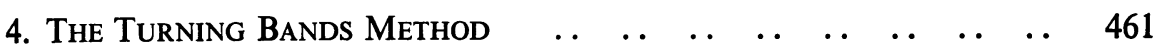

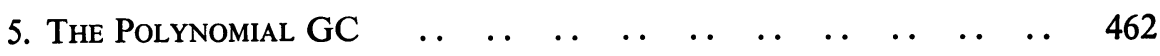

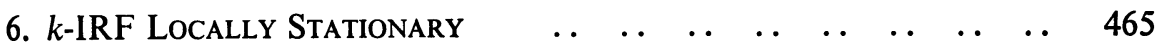

Received in revised form 22 March 1973. 


\section{INTRODUCTION}

The aim of the present study is essentially to act as a theoretical support to the optimum automatic contouring procedures being developed under the name of universal kriging, and which have been presented elsewhere in a more practical context $([7],[8],[9])$. The problem we are dealing with is the following. We know the values $z\left(x_{1}\right)$ taken by the physical variable of interest at several experimental locations $x_{i}$ (in general anywhere in twoor three-dimensional space) and we want to estimate at each point $x$ the value of the function $z$ (or of any other function deduced from it by a linear operation). We then assume that the function $z$ can be considered as a realization of an order-two random function $Z$, and we compute at each point $x$ the best linear predictor $z^{*}(x)$ of $z$ (in the sense of Wiener [12]). But for this the covariance function of $Z$ must be known. Statistical inference from a unique realization is in general reasonably possible in the stationary case (although some difficulties still arise when the experimental points are not located on a regular grid). Unfortunately, in numerous cases, this assumption of stationarity is physically inadmissible. Hence, it was necessary to find a wider class than that of order-two random functions, but one that would present the same advantages concerning statistical inference.

This class is that of intrinsic random functions (IRF) which constitutes, in fact, a particular case of the generalized stochastic processes with stationary increments of order $k$ defined by Guelfand and Vilenkin ([3], [4]), namely the case where the generalized processes are random functions (and not only distributions). This circumstance leads to special properties which deserve to be studied, especially because of their interest regarding applications. For instance, here the generalized covariances will be conditionally positive definite functions (and not only distributions). The aim is, moreover, to present IRF's as a generalization of stationary random functions (stationary, in this paper, is always taken in the sense of weak stationarity), and hence without referring to the theory of distributions.

- The simplest example of an IRF is that of a RF of the form $Y(x)=Y_{0}(x)$ $+P(x)$, when $Y_{0}(x)$ is a SRF and $P(x)$ a polynomial of degree $k$ with random coefficients. Besides, we will see that any IRF is, in a way, a limit of RF's of this type. The established concept of introducing this form of RF's comes naturally to mind when one tries to represent non-stationary phenomena. Indeed, it corresponds to the simplest hypothesis that can be made with a view to making statistical inference possible from a single realization, but IRF's present the same advantages concerning statistical inference, while offering a wider scope of possible models.

Realizations of an IRF of order 0, like Brownian motion, show characteristics that do not evoke the intuitive idea of stationarity (see, for example, 
[2], p. 87). This circumstance is amplified when the order $k$ of the IRF increases (see Figures $1,2,3$ ) so that the theory becomes applicable to larcer and larger ranges of non-stationary phenomena, while keep.ng, for the greatest part, the advantages linked to stationarity.

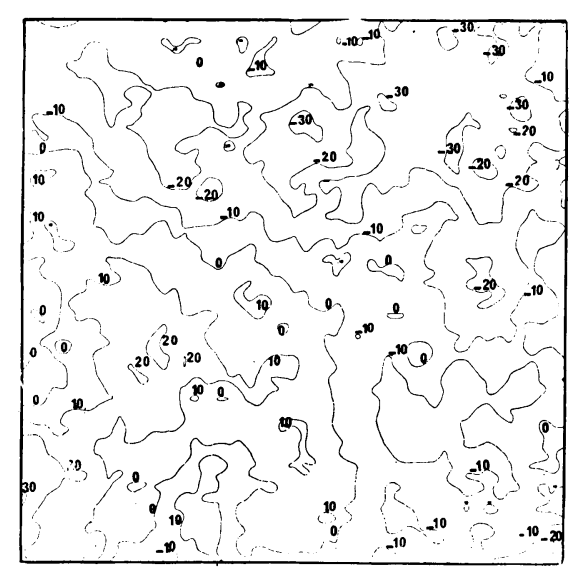

Figure 1

Realization of a 0-IRF with the GC $K(h)=-|h|$. The representation chosen vanishes at the center of the figure (from Orfeuil [10]).

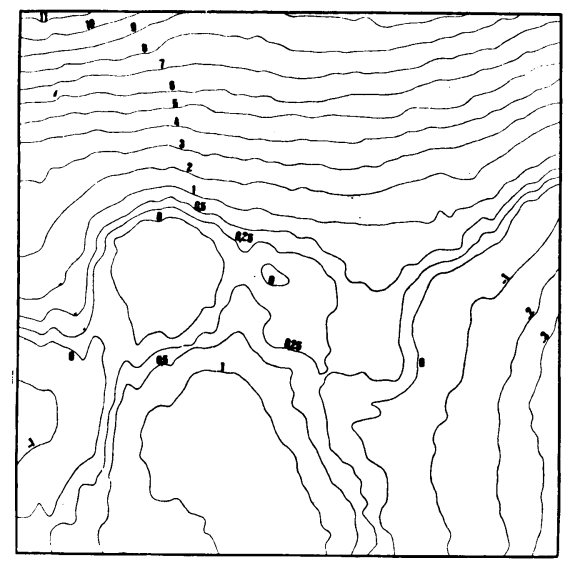

Figure 2

Realization of a 1-IRF with the GC $K(h)=-|h|^{3}$. The representation chosen, as well as its first derivatives, vanishes at the center of the figure (from Orfeuil [10]).

After giving the definitions and general properties of IRF's (Sections 1 and 2), we examine in Section 3 their generalized covariances, and the conditions that an IRF must fulfil to be differentiable, or identical to an IRF of lower order. The theory of the best linear intrinsic estimator (BLIE), given 


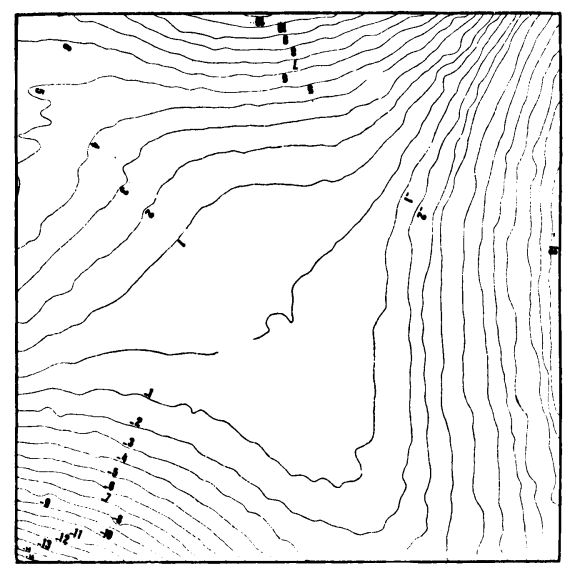

Figure 3

Realization of a 2-IRF with the GC $K(h)=-|h|^{5}$. The representation chosen, as well as its first and second derivatives, vanishes at the center of the figure (from Orfeuil [10]).

in Section 4, is a straightforward generalizaticn of Wiener s best linear predictor [12], and is fundamental for the applications, as is the turning bands method for simulating IRF s (Section 5). From a practical point of view, statistical inference is particularly easy to carry out by automatic procedures for a generalized covariance whose expression depends linearly on some unknown parameters. This is the justification for models with polynomial covariances (Section 6). These models are isotropic, but this is not so limiting as might be thought at first sight. The structure of an IRF is indeed determined by its generalized covariance only up to a random polynomial and, in practical cases, this implicit polynomial is often adequate to take into account the anisotropies of the real phenomenon. On the other hand, the IRF s with polynomial covariances are locally stationary, i.e., may be locally identified with a stationary RF up to a random polynomial. These circumstance enables us to define a precise and locally significant notion of trend, or drift, and this is also important in the applications (Section 7).

\section{Definition and General Properties}

\subsection{The spaces $\Lambda$ and $M_{c}$}

I shall denote by $\Lambda$ the vector space of real measures in $\boldsymbol{R}^{n}$ with finite supports. For any function $f$ on $\boldsymbol{R}^{n}$ and $\lambda \in \Lambda$, the integral $\int f(x) \lambda(d x)$ is thus a finite linear expression of the form $\sum_{i} \lambda_{i} f\left(x_{i}\right)$. If $Z: \boldsymbol{R}^{n} \rightarrow L^{2}(\Omega, \mathscr{A}, P)$ is a real order-two random function, it admits a linear extension $Z: \Lambda \rightarrow L^{2}(\Omega, \mathscr{A}, P)$ defined by putting $Z(\lambda)=\int \lambda(d x) Z(x), \lambda \in \Lambda$. The function $\lambda \rightarrow\|\lambda\|=\|Z(\lambda)\|$ 
is a norm on $\Lambda$ if $\int \lambda(d x) Z(x)$ implies $\lambda=0$, this condition being equivalent to the strict positivity of the covariance matrix $\left\langle Z\left(x_{\alpha}\right), Z\left(x_{\beta}\right)\right\rangle$ for any finite set of distinct points $x_{\alpha}$ in $\boldsymbol{R}^{n}$. Under this condition, $\Lambda$ is prehilbertian for the norm $\|\lambda\|$, and the completed space $\tilde{\Lambda}$ may be identified with the real Hilbert space $H \subset L^{2}(\Omega, \mathscr{A}, P)$ generated by the $Y(x), x \in R^{n}$. If the condition of strict positivity is not fulfilled, it is always posssible to take a convenient quotient-space instead of $\Lambda$ itself, and nothing is changed substantially.

It will often be useful in the sequel to consider another topology for $\Lambda$. Let $M_{c} \supset \Lambda$ be the space of measures with compact supports. $M_{c}$ is the exact dual of the space $\mathscr{C}$ of the continuous functions on $\boldsymbol{R}^{n}$ (for the compact convergence topology). We shall consider only the weak topology on $M_{c}$, and the corresponding relative topology on $\Lambda$. For this weak topology, the convergence $\mu_{n} \rightarrow \mu$ in $M_{c}$ is equivalent to the following two conditions:

(1) the sequence $\left\{\mu_{n}\right\}$ is weakly convergent towards $\mu$;

(2) the supports of the measures $\mu_{n}$ are contained in a fixed compact set.

The random function $Z: \boldsymbol{R}^{n} \rightarrow L^{2}(\Omega, \mathscr{A}, P)$ is then strongly continuous if and only if the mapping $\lambda \rightarrow Z(\lambda)$ is continuous on $\Lambda$ for the relative $M_{c}$ topology. The if part is obvious. Conversely, if $Z$ is strongly continuous on $\boldsymbol{R}^{n}$, the covariance function $\langle Z(x), Z(y)\rangle$ is continuous on $\boldsymbol{R}^{n} \times \boldsymbol{R}^{n}$. But the weak convergence $\lambda_{n} \rightarrow 0$ in $\Lambda \subset M_{c}$ implies $\lambda_{n} \otimes \lambda_{n} \rightarrow 0$ in $M_{c}\left(R^{n} \times R^{n}\right)$, and

$$
\left\|Z\left(\lambda_{n}\right)\right\|^{2}=\int \lambda_{n}(d x)\langle Z(x), Z(y)\rangle \lambda_{n}(d y) \rightarrow 0 .
$$

Thus, the mapping $\lambda \rightarrow Z(\lambda)$ is continuous.

If $Z$ is continuous on $\Lambda$, it admits a unique continuous extension on $M_{c}$, defined by putting $Z(\mu)=\int \mu(d x) Z(x)\left(\mu \in M_{c}\right)$. If $\|Z(x)\|=0$ implies $\mu=0$, $M_{c}$ may be identified with a subspace (generally not closed) of $\tilde{\Lambda}$, but the weak topology on $M_{c}$ is generally strictly stronger than the prehilbertian relative topology induced by $\tilde{\Lambda}$.

For any $h \in \boldsymbol{R}^{n}$ and $\mu \in M_{c}$, we define the translated measure $\tau_{h} \mu$ by

$$
\int \tau_{h}(d x) f(x)=\int \mu(d x) f(x+h)(f \in \mathscr{C}) .
$$

If $Z$ is a stationary random function (SRF), there exists a group of unitary operators $U_{h}, h \in \boldsymbol{R}^{n}$ on $H=Z(\tilde{\Lambda})$ satisfying $U_{h} Z(\lambda)=Z\left(\tau_{h} \lambda\right)(\lambda \in \Lambda)$. Moreover, if $Z$ is a continuous SRF, the group $U_{h}$ is continuous and we also have $U_{h} Z(\mu)=Z\left(\tau_{h} \mu\right)$ for $\mu \in M_{c}$. This property may be used as definition of the SRF and, with a slight modification, will lead us to the notion of IRF.

\subsection{Definition of the IRF}

Now let $\Lambda^{\prime}$ be a subspace of $\Lambda$ closed in $\Lambda$ for the relative $M_{c}$ topology. In other words, there exists a family $f^{l}, l \in L$ of continuous functions on 
$\boldsymbol{R}^{n}$ such that $\Lambda^{\prime}=\left\{\lambda: \lambda \in \Lambda, \int \lambda f^{l}=0, l \in L\right\}$, or $\Lambda^{\prime}=M^{\prime} \cap \Lambda$ if $M^{\prime}$ is the subspace of $M_{c}$ orthogonal to the family $f^{l}, l \in L$. Then, we shall say that a linear mapping

$$
Z: \Lambda^{\prime} \rightarrow L^{2}(\Omega, \mathscr{A}, P)
$$

is a generalized (order-two) random function on $\Lambda^{\prime}$. We denote $H(Z)$, or simply $H$, the closure of the range $Z\left(\Lambda^{\prime}\right)$ in $L^{2}(\Omega, \mathscr{A}, P)$. If $\|Z(\lambda)\|=0$ implies $\lambda=0$ for $\lambda \in \Lambda^{\prime}$, the completed space $\tilde{\Lambda}^{\prime}$ of $\Lambda^{\prime}$ for the norm $\|\lambda\|=\|Z(\lambda)\|$ may be identified with $H$ itself. If this condition is not fulfilled, $\tilde{\Lambda}^{\prime}$ will denote the completion of the convenient quotient space. If $Z$ is continuous on $\Lambda^{\prime}$ for the relative $M_{c}$ topology (continuous generalized random function), it admits a unique continuous extension $Z: M^{\prime} \rightarrow L^{2}\left(\Omega, \mathscr{A}, P\right.$ ), and $M^{\prime}$ (or a convenient quotient space) may be identified with a dense (but not closed) subspace of $\tilde{\Lambda}^{\prime}$. In order to generalize the notion of stationarity, we now suppose $\Lambda^{\prime}$ closed for translations (i.e., $\tau_{h} \lambda \in \Lambda^{\prime}$ for $h \in R^{n}$ and $\lambda \in \Lambda^{\prime}$ ), and we say that the generalized random function $Z: \Lambda^{\prime} \rightarrow L^{2}(\Omega, \mathscr{A}, P)$ is an intrinsic random function (IRF) on $\Lambda^{\prime}$ if the mapping $h \rightarrow Z\left(\tau_{h} \lambda\right)$ is a SRF for any $\lambda \in \Lambda^{\prime}$, i.e., if there exists a group of unitary operators $U_{h}, h \in R^{n}$ on $H=Z\left(\tilde{\Lambda}^{\prime}\right)$ satisfying $U_{h} Z(\lambda)=Z\left(\tau_{h} \lambda\right)$ for any $\lambda \in \Lambda^{\prime}$. Obviously, an IRF $Z$ is continuous on $\Lambda^{\prime}$ if and only if the mapping $(h, \lambda) \rightarrow Z\left(\tau_{h} \lambda\right)$ is continuous on $R^{n} \times \Lambda^{\prime}$. In this case, the group $U_{h}$ is continuous on $\boldsymbol{R}^{n}$, and we also have $U_{h} Z(\mu)=Z\left(\tau_{h} \mu\right)$ for any $\mu \in M^{\prime}$.

The subspace $M^{\prime} \subset M_{c}$ being closed for translations, so also is its orthogonal complement, i.e., the closed subspace of $\mathscr{C}$ generated by the functions $f^{l}, l \in L$. In particular, if $L$ is finite, this implies that the $f^{l}$ are exponential-polynomial functions. In what follows, we shall examine only the case where the $f^{l}$ functions are polynomials. More precisely: for $k$, an integar $\geqq 0$, we denote $M_{k}$ the subspace of $M_{c}$ defined by the condition $\mu \in M_{k}$ if $\mu \in M_{c}$ and

$$
\int x_{1}^{i_{1}} x_{2}^{i_{2}} \cdots x_{n}^{i_{n}} \mu(d x)=0
$$

for $i_{1}, \cdots, i_{n}$, integers $\geqq 0$, such that $i_{1}+\cdots+i_{n} \leqq k$. For brevity, we shall write $l$ instead of $\left(i_{1}, \cdots, i_{n}\right), l \leqq k$ instead of $i_{1}+\cdots+i_{n} \leqq k$ and $f^{l}(x)$ for $x_{1}^{i_{1}} x_{2}^{i_{2}} \cdots x_{n}^{i_{n}}$. The subspace $\Lambda \cap M_{k}$ (closed in $\Lambda$ ) will be denoted $\Lambda_{k}$, and we shall say that an IRF $Z$ on $\Lambda_{k}$ is an IRF of order $k$, or a $k$-IRF. If a $k$-IRF $Z$ is continuous, its unique continuous extension on $M_{k}$ will also be denoted by $Z$. In the sequel, we consider only continuous $k-I R F$ 's.

\subsection{Representations of a $k$-IRF}

If $Z$ is an IRF on a subspace $\Lambda^{\prime} \subset \Lambda$, we say that an order-two random function $Y: R^{n} \rightarrow L^{2}(\Omega, \mathscr{A}, P)$ is a representation of $Z$ if $Z(\lambda)=\int Y(x) \lambda(d x)$ 
for any $\lambda \in \Lambda^{\prime}$. In the sequel, we examine only the case of a continuous $k$-IRF $Z$. It is easy to find measures $\lambda_{l} \in \Lambda\left(l=i_{1}+i_{2}+\cdots+i_{n} \leqq k\right)$ such that

$$
\int \lambda_{l}(d x) f^{s}(x)=\delta_{l}^{s}
$$

$\left(s=\left(j_{1}, \cdots, j_{n}\right), j_{1}+\cdots+j_{n} \leqq k, \delta_{l}^{s}=0\right.$ for $l \neq s$ and $\delta_{l}^{s}=1$ if $\left.s=l\right)$. If $\delta_{x}$ is the Dirac measure at $x \in \boldsymbol{R}^{n}$, clearly $\left(\delta_{x}-f_{x}^{l} \lambda_{l}\right) \in \Lambda_{k}$. Then, the random function $Y(x), x \in \boldsymbol{R}^{n}$, defined by

$$
Y(x) \doteq Z\left(\delta_{x}-f_{x}^{l} \lambda_{l}\right)
$$

is a representation of $Z$. For, if $\lambda \in \Lambda_{k}$, i.e., $\int \lambda(d x) f^{l}(x)=0$, we also have $\lambda=\int \lambda(d x)\left[\delta_{x}-f_{x}^{l} \lambda_{l}\right] . Z$ being a linear mapping of $\Lambda_{k}$ into $H=Z\left(\tilde{\Lambda}_{k}\right)$, we may write

$$
Z(\lambda)=\int \lambda(d x) Z\left(\delta_{x}-f_{x}^{l} \lambda_{l}\right)=\int \lambda(d x) Y(x)
$$

and $Y(x)$ is a representation of the $k$-IRF $Z$.

Let $X(x)$ be another representation of $Z$. By the very definition, we may write

$$
Z(\lambda)=\int \lambda(d x) Y(x)=\int \lambda(d x) X(x) \text { for any } \lambda \in \Lambda_{k} .
$$

In particular, for $\lambda=\delta_{x}-f_{x}^{l} \lambda_{l}$, we find

$$
Y(x)=Y(x)-f_{x}^{l} \int \lambda_{l}(d y) Y(y)=X(x)-f_{x}^{l} \int \lambda_{l}(d y) X(y) .
$$

From this relationship, we conclude:

(a) The representation defined by the relationship (1.2) is characterized by

$$
\int \lambda_{l}(d x) Y(x)=0
$$

For, if another representation $X(x)$ satisfies (1.3), we find

$$
Y(x)=X(x)-f_{\lambda}^{l} \int \lambda_{l}(d y) X(y)=X(x) .
$$

(b) Any other representation $X(x)$ of $Z$ is of the form

$$
X(x)=Y(x)+A_{l} f^{l}(x)
$$

for random variables $A_{l} \in L^{2}(\Omega, \mathscr{A}, P)$ satisfying $A_{l}=\int \lambda_{l}(d x) X(x)$. Converse- 
ly, for any random variables $A_{l} \in L^{2}(\Omega, \mathscr{A}, P)$, the random function $X(x)$ defined by the relationship (2.4) is obviously a representation of $Z$, because

$$
\int \lambda(d x) X(x)=\int \lambda(d x) Y(x) \text { for any } \lambda \in \Lambda_{k} .
$$

This representation satisfies

$$
A_{l}=\int \lambda_{l}(d x) X(x)
$$

by (1.1) and (1.3). Thus, the relationships (1.2) and (1.4) give us the general form of all the representations of the $k$-IRF $Z$.

The $k$-IRF $Z$ being continuous on $\Lambda_{k}$ (for the relative $M_{c}$ topology), all its representations are continuous, and, conversely, if $Z$ admits a continuous representation, it is a continuous $k$-IRF.

\subsection{The translations formula}

Let $Z$ be a continuous $k$-IRF. Let us choose measures $\lambda_{l}$ satisfying (1.1), and consider the representation $Y(x)=Z\left(\delta_{x}-f_{x}^{l} \lambda_{l}\right)$. From the very definition of an IRF, we have

$$
U_{h} Y(x)=Z\left(\delta_{x+h}-f_{x}^{l} \tau_{h} \lambda_{l}\right)
$$

for $h, x \in \boldsymbol{R}^{n}$. This may be written as follows

$$
U_{h} Y(x)=Z\left(\delta_{x+h}-f_{x+h}^{l} \lambda_{l}\right)+Z\left(f_{x+h}^{l} \lambda_{l}-f_{x}^{l} \tau_{h} \lambda_{l}\right) .
$$

But $Z\left(\delta_{x+h}-f_{x+h}^{l} \lambda_{l}\right)=Y(x+h)$, and the relationship (1.3) implies

$$
\begin{aligned}
Z\left(f_{x+h}^{l} \lambda_{l}-f_{x}^{l} \tau_{h} \lambda_{l}\right) & =f_{x+h}^{l} \int \lambda_{l}(d y) Y(y)-f_{x}^{l} \int \lambda_{l}(d y) Y(y+h) \\
& =-f_{x}^{l} \int \lambda_{l}(d y) Y(y+h) .
\end{aligned}
$$

Thus we obtain the following translations formula

$$
\begin{aligned}
U_{h} Y(x) & =Y(x+h)-A_{l}(h) f^{l}(x), \\
A_{l}(h) & =\int \lambda_{l}(d y) Y(y+h) .
\end{aligned}
$$

From the relationship (1.6) we can obtain an important inequality. Because $\|Y(x)\|$ is continuous on $R^{n}$, and the measures $\lambda_{l} \in \Lambda$ have their supports included in the same compact, there exists a real $B>0$ such that

$$
|h| \leqq 1 \Rightarrow\left\|\int \lambda_{l}(d y) Y(x+h)\right\| \leqq B .
$$


By writing (1.6) in the form

$$
Y(x+h)=U_{h} Y(x)+f^{l}(x) \int \lambda_{l}(d y) Y(y+h),
$$

and taking into account the inequality $\left|f^{l}(x)\right| \leqq r^{l}$ (with $r=|x|$ and $l=i_{1}+\cdots+i_{n}=$ degree of the monomial $f^{l}$ ) we thus obtain for $|h| \leqq 1$ an inequality of the form

$$
\|Y(x+h)\| \leqq\|Y(x)\|+\sum_{i=0}^{k} b_{i} r^{i} \quad\left(b_{i} \geqq 0\right) .
$$

If now $u$ is a unit vector in $\boldsymbol{R}^{n}$ and $\varepsilon$ a real number with $0 \leqq \varepsilon \leqq 1$, we find by iteration

$$
\begin{aligned}
\|Y(u)\| & \leqq Y(0) \|+b_{0}, \\
\|Y(2 u)\| & \leqq Y Y(u) \|+\sum_{0}^{k} b_{i}, \\
\cdot & \cdot . \cdot . \cdot . \cdot . \cdot . \\
\|Y(m u)\| & \leqq\|Y[(m-1) u]\|+\sum_{0}^{k} b_{i}(m-1)^{i}, \\
\|Y[(m+\varepsilon) u]\| & \leqq\|Y(m u)\|+\sum_{0}^{k} b_{i} m^{i} .
\end{aligned}
$$

By adding these inequalities, we obtain an inequality of the form

$$
\|Y[(m+\varepsilon) u]\| \leqq \sum_{0}^{k+1} B_{i} m^{i} \leqq a+b(m+\varepsilon)^{k+1}
$$

for convenient $a, b$, real $\geqq 0$ independent of $u$ and $\varepsilon$. Thus, for any $x \in \boldsymbol{R}^{n}$, the representation $Y(x)$ of $Z$ satisfies the following inequality

$$
\|Y(x)\| \leqq a+b r^{k+1} \quad(r=|x|) .
$$

By (1.4), it is clear that any other representation of $Z$ satisfies inequalities of the same type.

From the relationship (1.7), we conclude that the integral $\int Y(x) \mu(d x)$ exists not only for any measure $\mu \in M_{c}$ with compact support, but also for any measure $\mu$ with a sufficiently rapid decrease at infinity. For instance, if $\phi \in \mathscr{S}$ is an infinitely differentiable function rapidly decreasing at infinity, as also do each of its derivatives, the regularized random function $Y_{\phi}(x)$ defined by

$$
Y_{\phi}(x)=\int \phi(y) Y(x+y) d y
$$


always exists, and is an infinitely differentiable random function, as can easily be verified.

Besides, we note that for any $\lambda \in \Lambda_{k}$, the integral $\int \lambda(d x) Y_{\phi}(x)$ does not depend on the choice of the particular representation $Y(x)$ of the $k$-IRF we have used, as shown by

$$
\begin{aligned}
\int \lambda(d x) Y_{\phi}(x) & =\int \phi(y) d y \int \lambda(d x) Y(x+y) \\
& =\int \phi(y) U_{y} Z(\lambda) d y .
\end{aligned}
$$

In other words, the regularized RF $Y_{\phi}(x)$ is a representation of the $k-I R F Z_{\phi}$ defined on $\Lambda_{k}$ by putting $Z_{\phi}(\lambda)=\int \phi(y) U_{y} Z(\lambda) d y$.

We shall say that the $k$-IRF $Z_{\phi}$ is the regularized function of $Z$ by $\phi$. Clearly, $Z_{\phi}$ is infinitely differentiable if $\phi \in \mathscr{S}$ (i.e., any representation of $Z_{\phi}$ is infinitely differentiable, or $Z_{\phi}(D)$ exists for any derivative $D$ ). If $D$ is a derivative of order $p$, the corresponding derivative $D Z_{\phi}$, defined by

$$
D Z_{\phi}(\lambda)=(-1)^{p} \int D \phi(y) U_{y} Z(\lambda) d y
$$

is a $(k-p)$-IRF for $p \leqq k$, and a SRF for $p>k$.

\subsection{A decomposition theorem}

If $Y(x), x \in R^{n}$ is a SRF, the mapping $\lambda \rightarrow \int \lambda(d x) Y(x)$ obviously defines a $k$-IRF on $\Lambda_{k}$, which may be denoted $\lambda \rightarrow Y(\lambda)$. It is thus possible to add a $k$-IRF $Z_{c}$ and the SRF $Y$ considered as a $k$-IRF. Thə sum $Z_{c}+Y$ is the $k$-IRF $Z$ defined by $Z(\lambda)=Z_{c}(\lambda)+\int \lambda(d x) Y(x), \lambda \in \Lambda_{k}$. Then we may state the following theorem.

Theorem 1.5. Any continuous $k-I R F Z$ is the sum of a SRF and of an infinitely differentiable $k-I R F$.

Proof. Let $p_{a} \in \mathscr{S}$ be the function defined by its Fourier transform

$$
\tilde{p}_{a}(u)=\left(1+\frac{1}{2} a|u|^{2}+\cdots+\frac{1}{2} k^{\prime-1} a^{k^{\prime}}|u|^{2 k^{\prime}}\right) \exp \left(-a|u|^{2}\right) \text { for } a>0
$$

and an integer $k^{\prime}>\frac{1}{2} k$. All the derivatives of $1-\tilde{p}_{a}$ up to order $2 k^{\prime}>k$ vanish in $u=0$. Thus, the measure $\delta-p_{a}(x) d x$ is in $M_{k}$. Put $Y_{a}(x)=Z\left(\delta_{x}-\tau_{x} p_{a}\right)$, i.e., $Y_{a}(x)=U_{x} Z\left(\delta-p_{a}\right)$. In other words, $Y_{a}(x)$ is a SRF. From the definition of the regularized $Z_{p_{a}}$, we then have $Z=Y_{a}+Z_{p_{a}}$, where $Y_{a}$ is the mapping $\lambda \rightarrow \int Y_{a}(x) \lambda(d x), \lambda \in \Lambda_{k}$, and the regularized $Z_{p_{a}}$ is an infinitely differentiable $k$-IRF. 
For any representation $Y(x)$ of $Z$, we have

$$
Y_{a}(x)=Z\left(\delta_{x}-\tau_{x} p_{a}\right)=Y(x)-\int p_{a}(y) Y(x+y) d y,
$$

because $\left(\delta_{x}-\tau_{x} p_{a}\right) \in \Lambda_{k}$, and the decomposition theorem gives simply $Y(x)=Y_{a}(x)+Y_{p_{a}}(x)$, where $Y_{a}(x)=Y(x)-Y_{p_{a}}(x)$ is a SRF and $Y_{p_{a}}(x)$ a representation of the infinitely differentiable $k$-IRF $Z_{p_{-}}$.

Also note the following corollary.

Corollary. For any $k-I R F$, there exists a sequence $\left\{Y_{n}\right\}$ of $S R F$ such that $\int \lambda(d x) Y_{n}(x)$ strongly converges toward $Z(\lambda)$ for any $\lambda \in \Lambda_{k}$.

With the preceding notation, we have to show that, for $\lambda \in \Lambda_{k}, \lim Y_{a}(\lambda)=Z(\lambda)$ for $a \rightarrow \infty$, i.e., $\lim \int p_{a}(y) U_{y} Z(\lambda) d y=0$. Let $\chi(d u)$ be the spectral measure associated with the SRF $U_{y} Z(\lambda)$. Then, by $\left|\tilde{p}_{a}\right|^{2} \leqq 1$ and $\left|\tilde{p}_{a}(u)\right|^{2} \rightarrow 0$ for any $u \in \boldsymbol{R}^{n}$, we obtain

$$
\left\|\int p_{a}(y) U_{y} Z(\lambda) d y\right\|^{2}=\int\left|\tilde{p}_{a}(u)\right|^{2} \chi(d u) \rightarrow 0,
$$

i.e., $Y_{a}(\lambda) \rightarrow Z(\lambda)$.

\subsection{Drift of an IRF}

Let $Z$ be a continuous $k$-IRF, $H_{0}$ the subspace of $H=H\left(\tilde{\Lambda}_{k}\right)$ containing the invariant elements of $H$ (i.e., $X \in H_{0}$ if $X \in H$ and $U_{h} X=X$ for any $h \in \boldsymbol{R}^{n}$ ) and $\Pi_{0}$ the projector on $H_{0}$ (in the ergodic case, $\Pi_{0}$ may be identified with the expected value). By putting $m_{0}(\lambda)=\Pi_{0} Z(\lambda)$ for $\lambda \in \Lambda_{k}$, we define a continuous $k$-IRF $m_{0}$ obviously invariant for the group $U_{h}$. Let us choose $\lambda \in \Lambda$ satisfying (1.1), and write $m_{0}(x)=m_{0}\left(\delta_{x}-f_{x}^{l} \lambda_{l}\right)$. Then, the representation $m_{0}(x)$ of the $k$-IRF $m_{0}$ is a polynomial of degree $\leqq k+1$ with coeffcients in $H_{0}$ (constant in the ergodic case).

Proof. If $Z$ is infinitely differentiable, so also are $m_{0}$ and its representation $m_{0}(x)$. By $m_{0}(x) \in H_{0}$ and the translations formula (1.6), we obtain $m_{0}(x)=m_{0}(x+h)-A_{l}(h) f^{l}(x)$. Differentiation of order $k+1$ with respect to $x$ eliminates the terms $A_{l}(h) f^{l}(x)$, and then differentiation of order 1 with respect to $h$ eliminates $m_{0}(x)$. Hence, all derivatives of order $k+2$ of $m_{0}(x)$ vanish identically, and $m_{0}(x)$ is a polynomial of degree $\leqq k+1$. The decomposition theorem shows that the result remains true when $Z$ is not differentiable.

The polynomial $m_{0}(x)$ with invariant coefficients depends on the choice of the representation we have used. But it is easy to verify that the terms of degree $k+1$ do not depend on it, and thus constitute a property of the $k$-IRF $Z$ itself. The corresponding homogeneous polynomial of degree $k+1$ with invariant coefficients will be called the drift (or intrinsic drift) of the $k$-IRF. 
If $m_{0}=0$, the $k$-IRF $Z$ is said to be without drift. Obviously, $Z$ is without drift if and only if $\Pi_{0} Y(x)$ is a polynomial of degree $\leqq k$ with invariant coefficients for a representation of $Z$ (and then, the same is true for any representation).

\section{The Generalized Covariances (GC)}

\subsection{The class of the GC of a $k$-IRF}

Let $Z$ be a continuous $k$-IRF. We say that a continuous and symmetric function $K$ on $R^{n}$ is a generalized covariance (GC) of $Z$ if

$$
\langle Z(\lambda), Z(\mu)\rangle=\int \lambda(d x) K(x-y) \mu(d y) \text { for } \lambda, \mu \in \Lambda_{k} .
$$

This is equivalent to the condition:

$$
\int \lambda(d x) K(x-y) \lambda(d y)=\|Z(\lambda)\|^{2} \quad\left(\lambda \in \Lambda_{k}\right) .
$$

The family of the continuous and symmetric functions $K$ satisfying the condition (2.1) will be called the class of the GC of $Z$. We will show that such GC always exist, and, if one of them is known, we obtain all the others by adding arbitrary even polynomials of degree $\leqq 2 k$. In other words, there is an existence theorem, and a uniqueness theorem, the latter up to an equivalence defined by the relationship $K_{1} \equiv K_{2}$ if $K_{1}-K_{2}$ is an even polynomial of degree $\leqq 2 k$.

We shall also say that a continuous, symmetric function $K$ on $\boldsymbol{R}^{n}$ is conditionally positive definite of order $k$ if

$$
\int \lambda(d x) K(x-y) \lambda(d y) \geqq 0 \text { for any } \lambda \in \Lambda_{k} .
$$

From the definition, the GC of a $k$-IRF (if any) are $k$-conditionally positive definite. Conversely, if $K$ is a continuous $k$-conditionally positive definite function, there exists a continuous $k$-IRF $Z$ such that the $Z(\lambda), \lambda \in \Lambda_{k}$ are Gaussian random variables satisfying (2.1), and these two classes of functions may be identified. The following theorem gives their characterization.

Theorem 2.1. Let $K$ be a continuous and symmetrical function on $R^{n}$. Then, $K$ is $k$-conditionally positive definite if and only if it admits the representation:

$$
K(h)=\int \frac{\cos (2 \pi(u h))-1_{B}(u) P_{k}(2 \pi(u h))}{\left(4 \pi^{2}|u|^{2}\right)^{k+1}} \chi_{0}(d u)+K_{0}(h),
$$

where $K_{0}$ is an even $k$-conditionally positive definite polynomial of degree 
$\leqq 2 k+2,1_{B}$ the indicator of a neighbourhood of the origin, and $\chi_{0}$ a positive measure, necessarily unique, without atom at the origin, and such that

$$
\int\left[1+4 \pi^{2}|u|^{2}\right]^{-k-1} \chi_{0}(d u)<\infty
$$

$\left(P_{k}\right.$ is the polynomial $\left.P_{k}(x)=\sum_{0}^{k}(-1)^{p} x^{2 p} /(2 p) !\right)$.

Proof. It follows from Section 1.4 that any representation of a $k$-IRF is a generalized stochastic process with stationary increments, so that the existence and uniqueness theorem as well as Theorem 2.1 are simple consequences of the Guelfand-Vilenkin theory ([3], Chapter 2, Section 4 and Chapter 3, Section 3). Note that it is also possible to derive them by spectral analysis, i.e., without using the distribution theory.

\section{$2.2 k$-IRF without drift}

Let $Z$ be a $k$-IRF, $K$ a GC of $Z$ which admits the representation (2.2), $\lambda \in \Lambda_{k}$ and $\tilde{\lambda}$ its Fourier transform. Then, it follows from (2.2) that the spectral measure $\chi_{\lambda}$ associated with the SRF $x \rightarrow U_{x} Z(\lambda)$ is

$$
\chi_{\lambda}=\left(|\tilde{\lambda}|^{2} / 4 \pi^{2}|u|^{2 k+2}\right) \chi_{0}+a \delta,
$$

and the atom $a$ is given by

$$
a=\int \lambda(d x) K_{0}(x-y) \lambda(d y) .
$$

In other words, $Z$ is without drift if and only if the degree of the polynomial $K_{0}$ is $\leqq 2 k$. More precisely, we may state the following theorem.

Theorem 2.2. Let $Z$ be a continuous $k$-IRF, $K$ a GC and $Y(x), x \in \boldsymbol{R}^{n}$ a representation of $Z$. Then, the following three conditions are equivalent:

(a) $Z$ is without drift (i.e., $\Pi_{0} Z=0$ ),

(b) $K(h) /|h|^{2 k+2} \rightarrow 0$ for $|h| \rightarrow \infty$,

(c) $Y(x) /|x|^{k+1} \rightarrow 0$ strongly for $|x| \rightarrow \infty$.

Proof. By the inequality $\left|\cos x-P_{k}(x)\right| \leqq x^{2 k+2} /(2 k+2)$ !, we have

$$
\left|\frac{\cos (2 \pi(u h))-P_{k}(2 \pi(u h))}{\left(4 \pi^{2} u^{2}\right)^{k+1}}\right| \leqq \frac{|h|^{2 k+2}}{(2 k+2) !}
$$

and, by the dominated convergence theorem

$$
\lim _{|h| \rightarrow \infty} \int_{B} \frac{\cos (2 \pi(u h))-P_{k}(2 \pi(u h))}{|h|^{2 k+2}\left(4 \pi^{2} u^{2}\right)^{k+1}} \chi_{0}(d u)=0 .
$$


Thus, the condition (b) is fulfilled if and only if the polynomial $K_{0}$ of Theorem 2.3 is of degree $\leqq 2 k$, i.e., if (a) is satisfied, and thus (a) and (b) are equivalent.

By the relationship (1.4), we may assume that the representation $Y(x)$ of $Z$ is of the form (1.2). Then, the covariance of $Y(x)$ is the function

$$
\begin{gathered}
\langle Y(x), Y(y)\rangle=K(x-y)-f^{l}(x) \int K(z-y) \lambda_{l}(d z)-f^{l}(y) \int K(z-x) \lambda_{l}(d z) \\
+f^{l}(x) f^{s}(y) \int \lambda_{l}(d z) K\left(z-z^{\prime}\right) \lambda_{l}\left(d z^{\prime}\right) .
\end{gathered}
$$

It follows from this relationship that (b) implies (c).

Finally, the representation $\Pi_{0} Y(x)$ of the drift $m_{0}=\Pi_{0} Z$ is a polynomial of degree $\leqq k+1$ with invariant coefficients. If the condition (c) is fulfilled, this polynomial is of degree $\leqq k$, and thus $Z$ is without drift.

\subsection{Examples}

By Theorem 2.1, a positive measure $\chi_{0}$ without atom at the origin and satisfying the condition (2.3) characterizes a $k$-conditionally positive definite function, and thus a possible model of a $k$-IRF.

For instance, the function $r^{\alpha}$ on $\boldsymbol{R}^{n}(r=|x|, \alpha$ real, $>0$ and different from an even integer) admits as a Fourier transform (in the sense of the distribution theory) the (pseudo) function $\pi^{-\alpha-\frac{1}{2} n} \Gamma\left[\frac{1}{2}(\alpha+n)\right] / \Gamma\left(-\frac{1}{2} \alpha\right) \rho^{-\alpha-n}$ (with $\rho=|u|)$. If $\alpha<2 k+2$, the absolutely continuous measure $\rho^{2 k+2-\alpha-n} d u$ satisfies the conditions of Thzorem 2.1. Thus $\Gamma\left(-\frac{1}{2} \alpha\right) r^{\alpha}$ is $k$-conditionally positive definite. If $\alpha=2 p+1(p \leqq k)$ is an odd integer, the function $(-1)^{p+1} r^{2 p+1}$ may be used as GC of a $k$-IRF. More generally, the function $K$ defined on $\boldsymbol{R}^{n}$ by

$$
K(r)=\sum_{p=0}^{k}(-1)^{p+1} a_{p} r^{2 p+1}
$$

is a $k$-GC if and only if the coefficients $a_{p}$ satisfy the condition

$$
\sum_{p=0}^{k} \frac{a_{p}}{\pi^{2 p+2+\frac{1}{2} n}} \frac{\Gamma\left[\frac{1}{2}(2 p+1+n)\right]}{\Gamma\left[1+\frac{1}{2}(2 p+1)\right]} \rho^{-n-2 p+1} \geqq 0
$$

for any $\rho>0$. These "polynomial isotropic" GC are interesting from the applications point of view, because their expression depends linearly on the coefficients $a_{p}$, an advantageous property for statistical inference. (See succeeding paragraph).

Now let us examine the case $n=1$. Let $X(x)$ be a SRF on $\boldsymbol{R}^{1}, \sigma(h)$ its stationary covariance and $\chi$ its spectral measure, and consider the successive integrals 


$$
Y_{0}(x)=\int_{0}^{x} X(\xi) d \xi, \cdots, Y_{k}(x)=\int_{0}^{x} Y_{k-1}(\xi) d \xi,
$$

or explicitly,

$$
Y_{k}(x)=\int_{0}^{x} \frac{(x-\xi)^{k}}{k !} X(\xi) d \xi
$$

Then, $Y_{k}$ is a representation of a $k$-IRF $Z_{k}$ with the $\mathrm{GC} K_{k}$ defined by

$$
K_{k}(h)=\int_{0}^{|h|} \frac{(|h|-\xi)^{2 k+1}}{(2 k+1) !} \sigma(\xi) d \xi .
$$

If $\Pi_{0} X(x)=0, Z_{k}$ is without drift, and the spectral measure $\chi_{0}$ of $X(x)$ is identical with the measure $\chi_{0}$ occurring in Theorem 2.3.

Now if $W_{0}(x)$ is a 0 -IRF with the GC $-|h|$ (for instance, $W_{0}$ may be a Brownian motion), the expression

$$
W_{k}(x)=\int_{0}^{x} \frac{(x-\xi)^{k-1}}{(k-1) !} W_{0}(\xi) d \xi
$$

constitutes a representation of a $k$-IRF with the GC $(-1)^{k+1}|h|^{2 k+1} /(2 k+1)$ !. More generally, if we put

$$
Y(x)=\sum_{p=0}^{k} b_{p} W_{p}(x)
$$

with arbitrary real coefficients $b_{p}$, we obtain a realization of a $k$-IRF which admits a polynomial GC of type (2.4). Conversely, it is possible to show that any $k$-IRF with a polynomial GC of type (2.4) admits a representation of the form (2.5).

\subsection{Applications of Theorem 2.1}

It follows from Theorem 2.1 that the classical results of the harmonic analysis may be applied to the $k$-IRF. Let $Z$ be a $k$-IRF without drift, and $\chi_{0}$ the spectral measure associated by Theorem 2.1 with its GC. Then, as we have seen, for any $\lambda \in \Lambda_{k}$, the SRF $x \rightarrow U_{x} Z(\lambda)$ admits the spectral measure $\chi_{\lambda}=\left(|\tilde{\lambda}|^{2} /\left(4 \pi^{2}|u|^{2}\right)^{k+1}\right) \chi_{0}(\tilde{\lambda}$ is the Fourier transform of $\lambda)$. Moreover, if we denote by $\zeta_{\lambda}$ the orthogonal random measure whose Fourier transform is $U_{x} Z(\lambda)$, the following relationship holds

$$
\tilde{\lambda}^{\prime} \zeta_{\lambda}=\tilde{\lambda} \zeta_{\lambda^{\prime}} \quad\left(\lambda, \lambda^{\prime} \in \Lambda_{k}\right)
$$

Furthermore, the mapping $\lambda \rightarrow \tilde{\lambda} /\left(4 \pi^{2}|u|^{2}\right)^{\frac{1}{2}(k+1)}$ may be extended by an isomorphism from the completion $\tilde{\Lambda}_{k}$ onto $L^{2}\left(\boldsymbol{R}^{n} / \chi_{0}\right)$, so that (2.6) remains valid for any $\lambda, \lambda^{\prime} \in \tilde{\Lambda}_{k}$. 
As a direct consequence, we see that the $k$-IRF $Z$ is differentiable up to order $p$ if and only if

$$
\int\left[1+4 \pi^{2}|u|^{2}\right]^{p-k-1} \chi_{0}(d u)<\infty .
$$

Thus, the integral

$$
K_{1}(h)=\int \frac{\cos (2 \pi(u h))-P_{k}(2 \pi(u h))}{\left(4 \pi^{2}|u|^{2}\right)^{k+1}} \chi_{0}(d u)
$$

exists, and represents a GC of $Z$, if land only if $Z$ is differentiable up to order $k$. If so, $K_{1}$ is the unique GC of $Z$ which vanishes at $h=0$ as also do its derivatives up to order $2 k$. In particular, any 0-IRF without drift admits a GC of the form

$$
K_{1}(h)=\int \frac{\cos (2 \pi(u h))-1}{4 \pi^{2}|u|^{2}} \chi_{0}(d u) .
$$

Any $k$-GC $K$ satisfies the inequality

$$
|K(0)-K(h)| \leqq a+b|h|^{2 k+2}
$$

for convenient constants $a, b \geqq 0$, as can be shown from (2.2) or (1.7). Then, the following criterion is easy to prove by harmonic analysis: a $k$-IRF is differentiable if and only if its GC satisfies inequalities of the form

$$
|K(0)-K(h)| \leqq \alpha|h|^{2}+\beta|h|^{2 k+2} \text {. }
$$

Let us now examine under which condition a $k$-IRF $Z$ actually is of order $<k$, i.e., is the restriction to $\Lambda_{k}$ of a $(k-1)$-IRF $Z$.

Theorem 2.4. Let $Z$ be a continuous $k$-IRF without drift, $k \geqq 1$ (respectively, a 0 -IRF such that $\Pi_{0} Z=0$ ). Then, the following three conditions are equivalent.

(a) $Z$ is the restriction to $\Lambda_{k}$ of a continuous $(k-1)$-IRF $Z$ (resp. the restriction to $\Lambda_{0}$ of a continuous SRF $Z$ ), and $Z$ is unique up to a drift (resp. to an invariant).

(b) There exists a measure $\chi_{0}^{\prime} \geqq 0$ without atom at the origin such that $\int\left[1+4 \pi^{2}|u|^{2}\right]^{-k} \chi_{0}^{\prime}(d u)<\infty$, and the spectral measure associated with $Z$ by Theorem 2.1 is $\chi_{0}=\left(4 \pi^{2}|u|^{2}\right) \chi_{0}^{\prime}$. If so, $\chi_{0}^{\prime}$ is the spectral measure associated with the $(k-1)$-IRF $Z$ (with the SRF $Z$ ).

(c) The GC's of $Z$ satisfy inequalities of the form $|K(h)| \leqq a+b|h|^{2 k}$ (resp. are bounded on $\boldsymbol{R}^{n}$ ).

Proof. Let $Z$ be a $k$-IRF and $Z, Z^{\prime}(k-1)$-IRF such that $Z(\lambda)=Z^{\prime}(\lambda)=Z(\lambda)$ for any $\lambda \in \Lambda_{k}$. Then, for any $\lambda^{\prime} \in \Lambda_{k-1}$, we find 


$$
\left(U_{h}-I\right) Z^{\prime}(\lambda)=Z\left(\tau_{h} \lambda-\lambda\right)=\left(U_{h}-I\right) Z(\lambda) .
$$

Thus, $Z^{\prime}(\lambda)=Z(\lambda)$ up to an invariant, and $Z$ is unique up to a drift.

The implications (a) $\Rightarrow(b)$ and (b) $\Rightarrow$ (c) are trivial. Let $K$ be a $k$-GC such that (c) holds, and $\chi_{0}$ its spectral measure. If $B$ is the neighbourhood of 0 such that (2.1) holds, and $C=B^{c}$ the complementary set, the measure $1_{c} \chi_{0}$ trivially satisfies (b), so that we may suppose $\chi_{0}=1_{B} \chi_{0}$, i.e., $Z$ is infinitely differentiable. Then, the function $K_{1}$ defined by (2.8) exists and is a GC of $Z$. Moreover, by (2.4) and (c), there exists a real $B<\infty$ such that

$$
\left|K_{1}(h)\right| \leqq B|h|^{2 k} \text {. }
$$

It remains to prove that (2.11) implies (b). We start from the obvious relationship

$$
\cos x-P_{k}(x)=(-1)^{k+1} \int_{0}^{x} \frac{(x-\xi)^{2 k-1}}{(2 k-1) !}[1-\cos \xi] d \xi
$$

which implies, for $r=|h|$ and $\alpha=h / r$,

$$
\begin{aligned}
\cos 2 \pi(u h) & -P_{k}(2 \pi(u h)) \\
= & (-1)^{k+1}(2 \pi(u \alpha))^{2 k} \int_{0}^{r} \frac{(r-\rho)^{2 k-1}}{(2 k-1) !}[1-\cos (2 \pi(u \alpha) \rho)] d \rho .
\end{aligned}
$$

By substituting in (2.8) we obtain

$$
(-1)^{k+1} K_{1}(\alpha r)=\int_{0}^{r} \frac{(r-\rho)^{2 k-1}}{(2 k-1) !} d \rho \int \frac{(u \alpha)^{2 k}}{\left(u^{2}\right)^{k}} \frac{1-\cos (2 \pi(u x) \rho)}{4 \pi^{2} u^{2}} \chi_{0}(d u) .
$$

Let $\Phi_{\alpha}$ be the Laplace transform of $(-1)^{k+1} K_{0}(\alpha r)$, i.e.,

$$
\Phi_{\alpha}(\lambda)=(-1)^{k+1} \int_{0}^{\infty} K_{1}(x r) e^{-\lambda r} d r \quad(\lambda>0) .
$$

By (2.12), the function $(-1)^{k+1} K_{1}(\alpha r)$ is the convolutive product in $\boldsymbol{R}_{+}$of two functions, the Laplace transforms of which are respectively $\lambda^{-2 k}$ and

$$
\int \frac{(u \alpha)^{2 k}}{\left(u^{2}\right)^{k}} \frac{\chi_{0}(d u)}{\lambda\left(\lambda^{2}+4 \pi^{2}(u \alpha)^{2}\right)} .
$$

Thus, we obtain

$$
\Phi_{\alpha}(\lambda)=\frac{1}{\lambda^{2 k+1}} \int \frac{(u \alpha)^{2 k}}{\left(u^{2}\right)^{k}} \frac{\chi_{0}(d u)}{\lambda^{2}+(2 \pi(u \alpha))^{2}} .
$$

On the other hand, the function $(-1)^{k+1} K_{0}(\alpha r)$ is $\geqq 0$ by $(2.8)$, and thus (2.11) implies the inequality $\Phi_{\alpha}(\lambda) \leqq B(2 k) ! / \lambda^{2 k+1}$. By (2.13) and $(u \alpha)^{2} \leqq u^{2} \alpha^{2}=u^{2}$, we obtain 


$$
\int \frac{(u \alpha)^{2 k}}{\left(u^{2}\right)^{k}} \frac{\chi_{0}(d u)}{\lambda^{2}+4 \pi^{2} u^{2}} \leqq B(2 k) ! .
$$

If $\alpha$ is the unit vector of the $u_{i}$ axis, this implies

$$
\int \frac{u_{i}^{2 k}}{\left(\sum u_{j}^{2}\right)^{k}} \frac{\chi_{0}(d u)}{\left(\lambda^{2}+4 \pi^{2} u^{2}\right)} \leqq B(2 k) ! .
$$

By the convexity relationship $\sum_{1}^{n} x_{i}^{n} \geqq n^{1-p}\left(\sum x_{i}\right)^{p}, x_{i}$ and $p \geqq 0$, we have $\left(\sum u_{i}{ }^{2}\right)^{k} \leqq n^{k-1} \sum u_{i}{ }^{2 k}$. Thus, by substituting in (2.14) and summing from $i=1$ to $i=k$, we find

$$
\int \frac{\chi_{0}(d u)}{\lambda^{2}+4 \pi^{2} u^{2}} \leqq n^{k}(2 k) ! B
$$

The decreasing family $\chi_{\lambda}=\chi_{0} /\left(\lambda^{2}+4 \pi^{2} u^{2}\right)$ being dominated, it follows that there exists a positive bounded measure $\chi_{0}^{\prime}=\lim \chi_{\lambda}$, for $\lambda \downarrow 0$. By the relationship $4 \pi^{2} u^{2} \chi_{\lambda}=\chi_{0}-\lambda^{2} \chi_{\lambda}$, we get $\chi_{0}=\lim 4 \pi^{2} u^{2} \chi_{\lambda}=4 \pi^{2} u^{2} \chi_{0}^{\prime}$. Thus, (b) is true.

It remains to prove (b) implies (a). Let us at first examine the case $k=0$. Let $Z$ be a 0 -IRF without drift, satisfying (b), i.e., admitting a GC of the form

$$
K(h)=\int[\cos 2 \pi(u h)-1] \chi_{0}^{\prime}(d u)
$$

for a bounded positive measure $\chi_{0}^{\prime}$ without atom at the origin. Let $\chi_{\lambda}=|\tilde{\lambda}|^{2} \chi_{0}^{\prime}$ and $\zeta_{\lambda}$ be the spectral measure and the orthogonal random measure associated with a $\lambda \in \Lambda_{k}$. The function $1 / \tilde{\lambda}$ belongs to $L^{2}\left(R^{n}, \chi_{\lambda}\right)$, and thus the integral

$$
Y(x)=\int[(\exp \{-2 i \pi(u x)\}) / \tilde{\lambda}(u)] \zeta_{\lambda}(d u)
$$

exists and defines an SRF $Y(x), x \in \boldsymbol{R}^{n}$, For any $\mu \in \Lambda_{k}$, we may write

$$
\int Y(x) \mu(d x)=\int(\tilde{\mu}(u) / \tilde{\lambda}(u)) \zeta_{\lambda}(d u)=\int \zeta_{\mu}(d u)=Z(\mu)
$$

by $\tilde{\mu} \zeta_{\lambda}=\tilde{\lambda} \zeta_{\mu}$. Then (a) is true.

Now let $Z$ be a $k$-IRF without drift, $k>0$, suppose (b) is true and prove (a). For $\lambda \in \Lambda_{k-1}$, put $Y_{\lambda}(x)=Z\left(\tau_{x} \lambda-\lambda\right)\left(x \in R^{n}\right) . Y_{\lambda}(x)$ is a representation of a 0 -IRF and satisfies the relationship

$$
\left\|Y_{\lambda}(x)\right\|^{2}=\int \frac{|\exp \{-2 i \pi(u x)\}-1|^{2}|\tilde{\lambda}|^{2}}{\left(4 \pi^{2} u^{2}\right)^{k}} \chi_{0}^{\prime}(d u) .
$$

The function $|\tilde{\lambda}|^{2} /\left(4 \pi^{2} u^{2}\right)^{k}$ being bounded on $R^{n} \backslash\{0\}$, and $\chi_{0}^{\prime}$ without atom at the origin, it follows that $\left\|Y_{\lambda}(x)\right\|$ is bounded on $R^{n}$. By the result which we have already proved for the $0-\mathrm{IRF}$, there exists a unique element $Y_{\lambda} \in H$ such that $Y_{\lambda}(x)=\left(U_{x}-I\right) Y_{\lambda}$ and $\Pi_{0} Y_{\lambda}=0$. The spectral measure associated 
with $Y_{\lambda}$ is $|\lambda|^{2} \chi_{0}^{\prime} /\left(4 \pi^{2} u^{2}\right)^{k}$. The continuity of the linear mapping $\lambda \rightarrow Y_{\lambda}$ from $\Lambda_{k-1}$ into $H$ is then easy to prove, so that this mapping is a continuous $(k-1)$-IRF. For $\lambda \in \Lambda_{k} \subset \Lambda_{k-1}$, obviously

$$
\left(U_{x}-I\right) Y_{\lambda}=Z\left(\tau_{x} \lambda\right)-Z(\lambda)=\left(U_{x}-I\right) Z(\lambda),
$$

and thus $Y_{\lambda}=Z(\lambda)$ up to an invariant. But this invariant is null, for $\Pi_{0} Y_{\lambda}=\Pi_{0} Z(\lambda)=0$, and the equality $Y_{\lambda}=Z(\lambda)$ holds. This achieves the proof of Theorem 2.3. As an immediate consequence, we may state the following corollary.

Corollary. A $(k+p)$-IRF (respectively a $(p-1)$-IRF) with $k \geqq 0, p \geqq 1$ is the restriction to $\Lambda_{k+p}\left(\Lambda_{p-1}\right)$ of a $k$-IRF (a SRF) if and only if one of its GC satisfies an inequality of the form $|K(h)| \leqq a+b|h|^{2 k+2}$ (resp. is bounded on $\boldsymbol{R}^{n}$ ).

\section{The Best Linear Intrinsic Estimators (BLIE)}

In practical applications, we generally have to interpret the concerned phenomenon as a realization of a certain representation $Y(x)$ of a $k$-IRF $Z$. Statistical inference is then reasonably possible as far as the GC of $Z$ are concerned. On the contrary, if only one realization of $Y(x)$ is available, it is entirely impossible to specify which particular representation of $Z$ is involved in the experimental data. Thus, only "authorized" integrals, i.e., integrals of the form $\int \lambda(d x) Y(x), \lambda \in \Lambda_{k}$ or $\lambda \in M_{k}$, may be assigned a computable variance (because they depend only on $Z$, and not on the choice of the particular representation $Y(x))$.

Then, if $Y_{0}$ is an element of the Hilbert space $H(Y)$ generated by the $Y(x)$, $x \in \boldsymbol{R}^{n}$, we shall say that another element $Y^{*} \in H(Y)$ is an intrinsic estimator of $Y_{0}$ if the difference $\left(Y^{*}-Y_{0}\right)$ is itself an authorized integral, or a strong limit of authorized integrals. For, in this case only, the variance of the "error" $\left(Y^{*}-Y_{0}\right)$ uniquely depends on $Z$ and not on the representation - and may be computed at least approximately. In this context, it is natural to develop a theory of the best linear intrinsic estimators (BLIE).

The element $Y_{0} \in H(Y)$ we have to estimate will be, for instance, the "value" $Y\left(x_{0}\right)$ of $Y(x)$ at a given point $x_{0} \in \boldsymbol{R}^{n}$, or the integral $\int p(d x) Y(x)$, where the measure $p$ is known, or a derivative of $Y(x)$ at a given point, and so on. Generally speaking, we consider the case $Y_{0}=\mathscr{L}(Y)$, where $\mathscr{L}$ is an element of the completed $\tilde{\Lambda}_{Y}$ of $\Lambda$ for the norm $\|\lambda\|=\|j \lambda(d x) Y(x)\|$ (or of the convenient quotient space, if $\|\lambda\|$ is not a norm). But the space $\tilde{\Lambda}_{Y}$ depends on the choice of the particular representation $Y(x)$, and not only on the $k$-IRF $Z$ itself, and in practice we do not know which particular representation is involved. For this reason, the operator $\mathscr{L}$ must be taken so that $\mathscr{L}(Y)$ is 
defined for any representation $Y$ of $Z$. In other words, by (1.4), the monomials $f^{l}$ of degree $\leqq k$ necessarily belong to the domain of $\mathscr{L}$. Or, which is the same, there exists in $\Lambda$ a sequence $\left\{\lambda_{n}\right\}$ such that $\int \lambda_{n}(d x) Y(x)$ strongly converges in $H(Y)$ towards the limit $\mathscr{L} Y$ for any representation $Y(x)$ of $Z$. In particular, this implies the numerical convergence of the sequences $\left\{\int \lambda_{n}(d x) f^{l}(x)\right\}$ towards limits denoted $\mathscr{L} f^{l}$. Instead of $\mathscr{L}(Y)=\lim \int \lambda_{n}(d x) Y(x)$, we shall use the symbolic notation $\mathscr{L}(Y)=\int \mathscr{L}(d x) Y(x)$ for brevity.

In order to estimate $\mathscr{L}(Y)$, we know, say, the elements $Y(x), x \in S$ belonging to a compact set $S$ (the set of the "experimental data"). In other words, the only possible estimators $Y^{*}$ are in the form $\int \lambda(d x) Y(x)$, for measures $\lambda \in \Lambda(S)$ (i.e., with finite supports included into $S$ ) and, more generally, strong limits of such elements, i.e., elements of the form $\int \mathscr{L}^{*}(d x) Y(x)$ for operators $\mathscr{L}^{*}$ with "support" in $S$. For $\lambda \in \Lambda(S)$, the element $\int \lambda(d x) Y(d x)$ is an intrinsic estimator of $\mathscr{L}(Y)$ if and only if $\lambda$ satisfies the "universality conditions"

$$
\int \lambda(d x) f^{l}(x)=\mathscr{L} f^{l}
$$

i.e., $\lambda \in \mathscr{L}+\tilde{\Lambda}_{k}$. Now, if a sequence $\left\{\lambda_{n}\right\}$ in $\Lambda(S) \cap\left(\mathscr{L}+\tilde{\Lambda}_{k}\right)$ is such that $\left\{\int \lambda_{n}(d x) Y(x)\right\}$ strongly converges in $H(Y)$ for a given representation $Y(x)$, it follows from the universality condition that the sequence $\left\{\int \lambda_{n}(d x) X(x)\right\}$ is also convergent for any other representation $X(x)$. In other words, the set of the operators $\mathscr{L}^{*}$ with support in $S$ and such that $Y^{*}=\mathscr{L}^{*}(Y)$ is an intrinsic estimator of $\mathscr{L}(Y)$ (i.e., the closure $\overline{\Lambda(S) \cap\left(\mathscr{L}+\widetilde{\Lambda}_{k}\right)}$ ) does not depend on the choice of the representation $Y(x)$ we have used to define the norm $\|\lambda\|=\left\|\int \lambda(d x) Y(x)\right\|$.

This result may be stated in another equivalent manner: if

$$
\lambda_{n} \in \Lambda(S) \bigcap\left(\mathscr{L}+\tilde{\Lambda}_{k}\right),
$$

i.e., $\mathscr{L}-\lambda_{n} \in \tilde{\Lambda}_{k}$, the element

$$
\int \mathscr{L}(d x) Y(x)-\int \lambda_{n}(d x) Y(x)=Z\left(\mathscr{L}-\lambda_{n}\right)
$$

does not depend on the choice of the representation $Y(x)$. In other words, the strong convergence $\int \lambda_{n}(d x) Y(x) \rightarrow \mathscr{L}^{*}(Y)$ for a particular representation $Y(x)$ is equivalent to the convergence $Z\left(\mathscr{L}-\lambda_{n}\right) \rightarrow Z\left(\mathscr{L}-\mathscr{L}^{*}\right)$ and thus implies $\int \lambda_{n}(d x) X(x) \rightarrow \mathscr{L}^{*}(X)$ for any other representation $X(x)$.

The variety $\Lambda(S) \bigcap\left(\mathscr{L}+\tilde{\Lambda}_{k}\right)$ is empty if it is not possible to find $\lambda \in \Lambda(S)$ with $\int \lambda(d x) f^{l}(x)=\mathscr{L} f^{l}$, i.e., if there exist coefficients $C_{l}$ such that $C_{l} f^{l}(x)=0$ for any $x \in S$ and $C_{l} \mathscr{L} f^{l} \neq 0$. For this reason, we shall always suppose the monomials $f^{l}$ linearly independent on $S$, i.e., $C_{l} f^{l}(x)=0$ for any $x \in S$ implies $C_{l}=0$, so that the variety containing the intrinsic estimators on $S$ will never be empty. 
In order to determine the BLIE, it remains to write that $Z\left(\mathscr{L}-\mathscr{L}^{*}\right)$ is the projection of 0 into the linear variety $Z\left(\mathscr{L}-\overline{\left.\Lambda(S) \cap\left(\mathscr{L}+\tilde{\Lambda}_{k}\right)\right)}\right.$, which is closed and non-empty, by the above considerations. We obtain the condition: $\left\langle Z\left(\mathscr{L}-\mathscr{L}^{*}\right), Z(\lambda)\right\rangle=0$ for any $\lambda \in \Lambda_{k}(S)$. This condition expresses that the continuous function $y \rightarrow \int\left(\mathscr{L}(d x)-\mathscr{L}^{*}(d x)\right) K(x-y)$ is orthogonal to $\Lambda_{k}(S)$, and thus coincides on $S$ with a linear combination $\mu_{l} f^{l}$ (i.e., a polynomial of degree $\leqq k)$. Finally, the operator $\mathscr{L}^{*}$ with support in $S$ associated with the BLIE $Y^{*}=\mathscr{L}^{*}(Y)$ is characterized by the following conditions:

$$
\begin{aligned}
\mathscr{L}^{*} f^{l} & =\mathscr{L} f^{l}, \\
\int \mathscr{L} *(d x) K(x-y) & =\int \mathscr{L}(d x) K(x-y)+\mu_{l} f^{l}(y) \text { for all } y \in S ;
\end{aligned}
$$

and the corresponding "estimation variance" is

$$
\begin{aligned}
\int(\mathscr{L}(d x) & \left.-\mathscr{L}^{*}(d x)\right) K(x-y)\left(\mathscr{L}(d y)-\mathscr{L}^{*}(d y)\right) \\
& =\int \mathscr{L}(d x) K(x-y) \mathscr{L}(d y)-\int \mathscr{L}(d x) K(x-y) \mathscr{L}^{*}(d y)+\mu_{l} \mathscr{L} f^{l} .
\end{aligned}
$$

\section{Example 3.1 (The finite case)}

If the set $S$ of "experimental data" is finite, the preceding discussion is greatly simplified, because we only have to consider estimators of the form $\int \lambda(d x) Y(x)$ with $\lambda \in \Lambda(S)$. Let $x_{\alpha}, \alpha=1,2, \cdots, N$ be the points of $S$, and write $Y_{\alpha}, f_{\alpha}^{l}, K_{\alpha \beta}$, etc., instead of $Y\left(x_{\alpha}\right), f^{l}\left(x_{\alpha}\right), K\left(x_{\alpha}-x_{\beta}\right)$, etc. Then, the BLIE on $S$ for $Y_{0}=\mathscr{L}(Y)$ is $Y^{*}=\lambda^{\alpha} Y_{\alpha}$, with coefficients $\lambda^{\alpha}$ satisfying the following system:

$$
\begin{aligned}
\lambda^{\alpha} f_{\alpha}^{l} & =\mathscr{L} f^{l}, \\
\lambda^{\alpha} K_{\alpha \beta} & =\int \mathscr{L}(d x) K\left(x-x_{\beta}\right)+\mu_{l} f_{\beta}^{l} .
\end{aligned}
$$

The BLIE $Y^{*}=\lambda^{\alpha} Y_{\alpha}$ always exists, the functions $f^{l}$ being independent on $S$, so that the system (3.2) admits solutions. The unicity of $Y^{*}$ does not imply the regularity of the system (3.2), but only the relationship $\lambda_{1}^{\alpha} Y_{\alpha}=\lambda_{2}^{\alpha} Y_{\alpha}$ a.s. if $\lambda_{1}$ and $\lambda_{2}$ are two distinct solutions. As a matter of fact, the difference $v=\lambda_{1}-\lambda_{2}$ between two solutions will satisfy the system:

$$
v^{\alpha} f_{\alpha}^{l}=0, \quad v^{\alpha} K_{\alpha \beta}=\mu_{l}^{\prime} f_{\beta}^{l},
$$

from which it follows $\left\|v^{\alpha} Y_{\alpha}\right\|^{2}=v^{\alpha} K_{\alpha \beta} v^{\beta}=\mu_{l}^{\prime} f_{\beta}^{l} v^{\beta}=0$. It is not difficult to show that the system (3.2) is regular if and only if the following two conditions are fulfilled: 
(a) the matrix $K_{\alpha \beta}$ is strictly conditionally positive definite,

(b) the functions $f^{l}$ are linearly independent on $S$ (i.e., $C_{l} f_{\alpha}^{l}=0 \Rightarrow C_{l}=0$ ).

\section{Example 3.2 (Estimation of a drift)}

We suppose now that the $k$-IRF $Z$ admits as representation a SRF $Z(x)$ $=U_{x} Z_{0}$ with $\Pi_{0} Z_{0}=0$. By the corollary of Theorem 2.4 , it is the case if and only if $Z$ is without drift and admits a bounded GC, say $\sigma$.We may suppose that $\sigma$ is the stationary covariance of the $\operatorname{SRF} Z(x)=U_{x} Z_{0}$. Any other representation of $Z$ is of the form

$$
Y(x)=Z(x)+A_{l} f^{l}(x)
$$

where the $A_{l}$ are order-two random variables. In the present context, we shall say that the (random) polynomial $A_{l} f^{l}$ is the drift of the RF $Y(x)$. We may ask if it is possible to get a BLIE for the drift value $A_{l} f^{l}(x)$ at a given point $x$, and for the coefficients $A_{l}$ themselves. This new formulation of an old problem (see, for instance, [5]) will remain valid if $Z$ is only locally stationary (see Section 7), but not stationary, so that it will be possible to define a locally significant notion of a drift and to get a BLIE for it.

The first question that arises is whether $A_{l} \in H(Y)$ and $A_{l}=\mathscr{L}_{l} Y$ for operators $\mathscr{L}_{l}$ including the monomials $f^{s}$ in their domains. For this purpose, take $\lambda_{l} \in \Lambda$ satisfying the "universality condition" $\int \lambda_{l}(d x) f^{s}(x)=\delta_{l}^{s}$ ( $=0$ if $l \neq s$, $=1$ if $l=s$ ), and denote $\phi_{l}$ the density of the Gaussian centered law with variance $t$. The regularized functions

$$
\phi_{l}(x, t)=\int \lambda_{l}(d y) \phi_{t}(x+y)
$$

still satisfy the universality conditions

and we get

$$
\int \phi_{l}(x, t) f^{s}(x) d x=\delta_{l}^{s},
$$

$$
\int \phi_{l}(x, t) Y(x) d x=\int \phi_{l}(x, t) Z(x) d x+A_{l} .
$$

For $t \rightarrow \infty$, the ergodic theorem asserts that $\int \phi_{l}(x, t) Z(x) d x$ strongly converges towards 0 . Thus, the operators $\mathscr{L}_{l}$ defined by

$$
\mathscr{L}_{l}(Y)=\lim _{t \rightarrow \infty} \int \phi_{l}(x, t) Y(x) d x
$$

satisfy $\mathscr{L}_{l}(Y)=A_{l}$ for any representation $Y$, as required, and in particular $\mathscr{L}_{l} f^{s}=\delta_{l}^{s}$. 
Thus, there exists a BLIE for $A_{l}=\mathscr{L}_{l} Y$, say $A_{l}^{*}=\mathscr{L}_{l}^{*}(Y)$. The operator $\mathscr{L}_{l}^{*}$ (with support in the compact set $S$ of experimental data) is characterized by the system (3.1) written with $\sigma$ instead of $K$. But, in the second relationship (3.1), the term $\int \mathscr{L}_{l}(d x) \sigma(x-y)$ vanishes, by the ergodic theorem. Finally, we get the following system:

$$
\begin{aligned}
& \int \mathscr{L}_{l}^{*}(d x) f^{s}(x)=\delta_{l}^{s}, \\
& \int \mathscr{L}_{l}^{*}(d x) \sigma(x-y)=\mu_{l s} f^{s}(y) \text { for all } y \in S .
\end{aligned}
$$

The matrix $\left(\mu_{l s}\right)$ of the "Lagrange parameters" admits a very simple interpretation: it is identical with the covariance matrix $\left\langle A_{l}-A_{l}^{*}, A_{s}-A_{s}^{*}\right\rangle$, as is easy to verify by direct calculation. It is also easy to show that, for a given $x \in \boldsymbol{R}^{n}, A_{l}^{*} f^{l}(x)$ is the BLIE for the drift value in $x, A_{l} f^{l}(x)$.

Let $H_{Y}(S)$ be the Hilbert space generated by the $Y(x), x \in S$, and $H_{k}(S) \subset H_{Y}(S)$ the Hilbert space generated by the $\int \lambda(d x) Y(x)=Z(\lambda), \lambda \in \Lambda_{k}(S)$. By the system (3.3), the $A_{l}^{*}$ belong to the orthogonal $H_{k}^{\perp}(S)$ of $H_{k}(S)$ in $H_{Y}(S)$. Conversely, if $Y_{0} \in H_{k}^{\perp}(S)$, this element is of the form $Y_{0}=C^{l} A_{l}^{*}$. For, if $x \in S$, the relationship $Y(x)=Z\left(\delta_{x}-f_{x}^{l} \mathscr{L}_{l}^{*}\right)+A_{l}^{*} f^{l}(x)$ implies $\left\langle Y_{0}, Y(x)\right\rangle=$ $\left\langle Y_{0}, A_{l}^{*}\right\rangle f^{l}(x)$, and the projection of $Y_{0}$ into the orthogonal of the $A_{l}$ vanishes. Thus, $H_{k}^{\perp}(S)$ is spanned by the $A_{l}^{*}$. We shall say that $H_{k}^{\perp}(S)$ is the space of the drift and $H_{k}(S)$ the space of the residuals.

Then, by relationship $Y(x)=Z\left(\delta_{x}-f^{l}(x) \mathscr{L}_{l}^{*}\right)+A_{l}^{*} f^{l}(x)$, we conclude that for $x \in S$ the BLIE $A_{l}^{*} f^{l}(x)$ of the drift value at $x \in S$ is the projection of $Y(x)$ itself into the drift space $H_{k}^{\perp}(S)$. But this result does not remain true for $x \notin S$.

\section{The Turning Bands Method}

In practical applications, one must sometimes simulate realizations of a given random function in $\boldsymbol{R}^{2}$ or $\boldsymbol{R}^{3}$, and we will now describe a procedure which may be used in the isotropic case for SRF as well as for $k$-IRF.

First, let $t \rightarrow Y(t)$ be a SRF on $\boldsymbol{R}^{1}, C_{1}$ its covariance, and $s$ a unit vector in $\boldsymbol{R}^{n}$. Then, $x \rightarrow Z_{s}(x)=Y(\langle x, s\rangle)$ is a SRF on $\boldsymbol{R}^{n}$ and its covariance is defined by $\left\langle Z_{s}(x), Z_{s}(y)\right\rangle=C_{1}(\langle(x-y), s\rangle)$. If now $s$ is substituted by the unit random vector, $Z_{s}(x)$ is changed into a SRF $Z(x)$, the covariance $C$ of which is defined by

$$
C(h)=\int C_{1}(\langle h, s\rangle) \varpi_{n}(d s)
$$

where $\varpi_{n}$ is the probability concentrated on the unit sphere and invariant 
under rotations. Clearly, the covariance $C$ is isotropic, i.e., there exists a function $C_{n}$ on $\boldsymbol{R}_{+}$such that $C(h)=C_{n}(|h|)$, say explicitly

$$
C_{n}(r)=2 \Gamma\left(\frac{1}{2} n\right) \pi^{-\frac{1}{2}}\left(\Gamma\left[\frac{1}{2}(n-1)\right]\right)^{-1} \int_{0}^{1} C_{1}(v r)\left(1-v^{2}\right)^{\frac{1}{2}(n-3)} d v
$$

Let $G$ be the positive measure on $\boldsymbol{R}_{+}$such that

$$
C_{1}(r)=\int_{0}^{\infty} \cos 2 \pi \rho r G(d \rho)
$$

An easy calculation gives

$$
C_{n}(r)=2^{\frac{1}{2} n-1} \Gamma\left(\frac{1}{2} n\right) \int_{0}^{\infty}(2 \pi \rho r)^{1-\frac{1}{2} n} J_{\frac{1}{2} n-1}(2 \pi \rho r) G(d \rho)
$$

where $J_{\frac{1}{2} n-1}$ is the classical Bessel function. But we know from the Bochner theorem that (4.2) is the general form of an isotropic covariance on $\boldsymbol{R}^{n}$, so that the mapping $C_{1} \rightarrow C_{n}$ is one-to-one from the covariances on $R^{1}$ onto the isotropic covariances on $\boldsymbol{R}^{n}$. In other words, for any isotropic covariance $C_{n}$, there exists a unique covariance $C_{1}$ on $R^{1}$ such that (4.1) holds. For instance, if $n=3$, we find $C_{3}(r)=\int_{0}^{1} C_{1}(v r) d v$, and, conversely, $C_{1}(r)=d r C_{3}(r) / d r$.

If an isotropic covariance $C_{n}$ on $\boldsymbol{R}^{n}$ is given, the procedure described above (i.e., the "turning bands" method) will yield a realization of a SRF with covariance $C_{n}$, if it is applied to a realization of a SRF $Y$ with the corresponding covariance $C_{1}$ on $R^{1}$.

The turning bands method may also be used in order to construct representations of a $k$-IRF with a given isotropic GC on $\boldsymbol{R}^{\boldsymbol{n}}$, for it is easy to see from Theorem 2.1 that the relationship (4.1) defines a one-to-one mapping from the $k$-GC on $\boldsymbol{R}^{1}$ onto the isotropic $k$-GC on $\boldsymbol{R}^{n}$. Moreover, the monomials $r^{\alpha}, \alpha \geqq 0$ are eigen-functions for the turning bands operator (4.1), so that the polynomial isotropic GC on $\boldsymbol{R}^{n}$ (see Section 5 below) are generated by the polynomial GC on $\boldsymbol{R}^{1}$. This procedure was used by Orfeuil [10], who constructed the originals of the Figures 1,2 and 3 above. These figures give realizations of IRF order $k=0,1,2$ with GC proportional to $-|h|,|h|^{3}$ and $-|h|^{5}$ respectively. The representations chosen by Orfeuil vanish at the center of the figures, as well as their derivatives up to order $k$, and this explains the feature of Figure 3 in which nothing resembles an isotropic and stationary phenomenon any longer.

\section{The Polynomial GC}

Let us denote by $T_{n}$ the turning bands operator defined in (4.1), so that for any $\alpha>0$ the function $r \rightarrow r^{\alpha}$ is an eigen-function for $T_{n}$. If $\alpha=2 p+1$ is an odd integer, we find explicitly 


$$
T_{n} r^{2 p+1}=B_{n p} r^{2 p+1}, \quad B_{n, p}=p ! \Gamma\left(\frac{1}{2} n\right) /\left(\pi^{\frac{1}{2}} \Gamma\left(p+\frac{1}{2}(1+n)\right)\right) .
$$

As noted above, the function $K_{1}$ defined by

$$
K_{1}(r)=\sum_{p=0}^{k}(-1)^{p+1} a_{p} r^{2 p+1} /(2 p+1) !
$$

is a $k$-GC on $\boldsymbol{R}^{1}$ if and only if the function

$$
K_{n}(r)=\sum_{p=0}^{k}(-1)^{p+1} a_{p} B_{n p} r^{2 p+1} /(2 p+1) !
$$

is itself an isotropic $K$-GC on $\boldsymbol{R}^{n}$. With the notations (5.1) and (5.2), the conditions the coefficients $a_{p}$ have to satisfy do not depend on the dimension $n$.

From the relationship

$$
(-1)^{p+1} \frac{r^{2 p+1}}{(2 p+1) !}=4 \int_{0}^{\infty} \frac{\cos (2 \pi \rho r)-P_{p}(2 \pi \rho r)}{\left(4 \pi^{2} \rho^{2}\right)^{p+1}} d \rho,
$$

it follows that the function $K_{1}$ defined in (5.1) admits (up to an even polynomial of degree $\leqq 2 k$ ) the representation

$$
\begin{gathered}
K_{1}(r)=\int_{0}^{\infty} \frac{\cos 2 \pi \rho r-1_{B}(\rho) P_{k}(2 \pi \rho r)}{\left(4 \pi^{2} \rho^{2}\right)^{k+1}} g(\rho) d \rho, \\
g(\rho)=4 \sum_{p=0}^{k} a_{p}\left(4 \pi^{2} \rho^{2}\right)^{k-p} .
\end{gathered}
$$

In other words, $K_{1}$ (and $K_{n}$ ) are $k$-GC if and only if the polynomial $\Pi(x)=\Sigma a_{p} x^{2(k-p)}$ is $\geqq 0$ for $x$ real.

Lemma 5. An even polynomial $\Pi$ with real coefficients and degree $2 k$ satisfies $\Pi(x) \geqq 0$ for $x$ real if and only if there exists a polynomial $\Phi$ with real coefficients and degree $k$ such that $\Pi(x)=|\Phi(i x)|^{2}$.

Proof. The if part is obvious. Conversely, let $\Pi$ be an even polynomial with real coefficients and degree $2 k$ such that $\Pi(x) \geqq 0$ for $x$ real. Then (up to a positive multiplicative constant) $\Pi(z)$ is the product of terms of the type

$$
(z+i b)(z-i b)=(b+i z)(b-i z)
$$

with $b$ real, or

$$
(z-a)(z+a)(z-\bar{a})(z+\bar{a})=(\alpha+i z)(\bar{\alpha}+i z)(\alpha-i z)(\bar{\alpha}-i z),
$$

with $\alpha=i a$ and $a=a_{1}+i a_{2}, a_{1}, a_{2}$ real and $a_{1} \neq 0$. Each of these terms is of the form $\phi(i z) \phi(-i z)$ for a polynomial $\phi$ with real coefficients. The lemma follows. 
Let us now give the general form of the $k-\mathrm{IRF}$ with polynomial GC on $\boldsymbol{R}^{\mathrm{I}}$.

Theorem 5. A $k$-IRF $Z$ on $\boldsymbol{R}^{1}$ admits a polynomial GC if and only if it admits a representation $Y$ such that

$$
Y(x)=b_{0} W(x)+b_{1} \int_{0}^{x} W(\xi) d \xi+\cdots+b_{k} \int_{0}^{x} \frac{(x-\xi)^{k-1}}{(k-1) !} W(\xi) d \xi
$$

where the $b_{p}$ are real coefficients and $W(x)$ a representation of a 0-IRF with the $\mathrm{GC} K(h)=-|h|$.

Proof. If $Z$ admits the representation (5.3),

$$
X(x)=\int_{0}^{x}(x-\xi)^{k-1} W(\xi) /(k-1) ! d \xi
$$

is a representation of a $k$-IRF with the GC $(-1)^{k+1}|h|^{2 k+1} /(2 k+1)$ !. If $D^{p}$ denotes the derivation order $p$, we have

$$
Y(x)=\left(\Sigma b_{p} D^{k-p}\right) X(x)
$$

and the $k$-IRF $Z$ admits the GC

$$
K(h)=\left(\Sigma b_{p} D^{k-p}\right)\left(\Sigma(-1)^{k-p} D^{k-p}\right)|h|^{2 k+1} /(2 k+1) !
$$

i.e., a polynomial GC.

Conversely, let $Z$ be a $k$-IRF on $\boldsymbol{R}^{1}$ admitting a GC $K(h)$ of the form (5.1). From Lemma 5 and the preceding considerations we may write (up to an even polynomial of degree $\leqq 2 k$ )

$$
\begin{aligned}
& K(h)=2 \int_{-\infty}^{\infty} \frac{\cos 2 \pi u h-1_{B}(u) P_{k}(2 \pi u h)}{\left(4 \pi^{2} u^{2}\right)^{k+1}}|\Phi(2 i \pi u)|^{2} d u, \\
& \Phi(u)=\sum_{p=0}^{k} b_{p} u^{k-p},
\end{aligned}
$$

with convenient real coefficients $b_{p}$. Now let $\alpha \in \tilde{\Lambda}_{k}$ be the function defined by its Fourier transform $\tilde{\alpha}(u)=(2 i \pi u)^{k} \exp \left(-u^{2}\right)$, and $\zeta_{\alpha}$ the random orthogonal measure such that $Z\left(\tau_{h} \alpha\right)=\int \exp (-2 i \pi u h) \zeta_{\alpha}(d u)$. The corresponding spectral measure $\chi_{\alpha}$ is

$$
\chi_{\alpha}(d u)=2\left[|\tilde{\alpha}|^{2}|\Phi(2 i \pi u)|^{2} /\left(4 \pi^{2} u^{2}\right)^{k+1}\right] d u,
$$

and, for any $\lambda \in \Lambda_{k}$ the Fourier transform of which is $\tilde{\lambda}, \tilde{\lambda} / \tilde{\alpha} \in L^{2}\left(\boldsymbol{R}^{1}, \chi_{\alpha}\right)$ implies

$$
Z(\lambda)=\int(\tilde{\lambda}(u) / \tilde{\alpha}(u)) \zeta_{\alpha}(d u)
$$

The function $(\exp (-2 i \pi u x)-1)(-2 i \pi u)^{k} /[\tilde{\alpha}(u) \Phi(-2 i \pi u)]$ is also in $L^{2}\left(\boldsymbol{R}^{1}, \chi_{\alpha}\right)$, and thus there exists a random function $W(x)$ defined by 


$$
W(x)=\int_{-\infty}^{+\infty} \frac{(\exp \{-2 i \pi u x\}-1)(-2 i \pi u)^{k}}{\tilde{\alpha}(u) \Phi(-2 i \pi u)} \zeta_{\alpha}(d u)
$$

If $\lambda \in \Lambda_{0}$, we have

$$
\int \lambda(d x) W(x)=\int_{-\infty}^{+\infty} \tilde{\lambda}(u)(-2 i \pi u)^{k} /(\tilde{\alpha}(u) \Phi(-2 i \pi u)) \zeta_{\alpha}(d u)
$$

and thus

$$
\begin{aligned}
\left\|\int \lambda(d x) W(x)\right\|^{2} & =\int \frac{|\tilde{\lambda}|^{2}\left(4 \pi^{2} u^{2}\right)^{k}}{|\tilde{\alpha}|^{2}|\Phi(-2 i \pi u)|^{2}} \chi_{\alpha}(d u) \\
& =2 \int\left(|\tilde{\lambda}|^{2} / 4 \pi^{2} u^{2}\right) d u .
\end{aligned}
$$

From this relationship, it follows that $\lambda \rightarrow \int \lambda(d x) W(x)$ is a 0 -IRF with the GC

$$
2 \int_{-\infty}^{+\infty} \frac{\cos 2 \pi u h-1}{4 \pi^{2} u^{2}}=-|h| \text {. }
$$

Now let $Y(x)$ be defined by (5.3) and (5.5), and $\mu \in \Lambda_{k}$. By (5.5), we may write

$$
\begin{aligned}
\int \mu(d x) Y(x) & =\int \frac{\tilde{\mu}(u)(-2 i \pi u)^{k} \Sigma b_{p} /(-2 i \pi u)^{p}}{\tilde{\alpha}(u) \Phi(-2 i \pi u)} \zeta_{\alpha}(d u) \\
& =\int(\tilde{\mu}(u) / \tilde{\alpha}(u)) \zeta_{\alpha}(d u) .
\end{aligned}
$$

Thus, by (4.4), we have $\int \mu(d x) Y(x)=Z(\mu)$, and $Y(x)$ is a representation of $Z$. This completes the proof.

It is very easy to construct realizations of a 0 -FAI with GC $-|h|$ on $\boldsymbol{R}^{\mathbf{1}}$. For instance, we may choose a Wiener-Lévy process (Brownian motion). By Theorem 5, we are thus able to construct realizations of a $k$-IRF with a given polynomial GC, and by the turning bands method we may also obtain realizations of a $k$-IRF with a given polynomial covariance isotropic on $\boldsymbol{R}^{n}$.

\section{6. $k$-IRF LoCALLy STATIONARY}

Any stationary covariance is also a $k$-GC, and thus there exist $k$-IRF's which admit stationary representations and may be called stationary $k$-IRF's. Any representation of a stationary $k$-IRF is then of the form $Y(x)=Y_{0}(x)+P(x)$, where $Y_{0}(x)$ is a SRF and $P(x)$ a polynomial of degree $\leqq k$ with random coefficients $A_{l}$. From Theorem 2.4, we know that a $k$-IRF is stationary if and only if it admits a GC bounded on $\boldsymbol{R}^{n}$. This condition is never fulfilled by a polynomial GC. But, on the other hand, we shall see that the $k$-IRF with polynomial GC are always locally stationary in the following sense. 
Definition. A $k$-IRF $Z$ is locally stationary if there exist a bounded open set $V \subset R^{n}$ and a representation $Y(x)$ of $Z$ such that $Y(x)$ coincides on $V$ with a $\operatorname{SRF} Y_{V}(x)$.

Clearly, if $Z$ is locally stationary on an open set $V$, it is still so on any translation $V^{\prime}$ of $V$, but the SRF $Y_{V^{\prime}}$ cannot be identical to $Y_{V}$ if $Z$ itself is not stationary. This property is important for practical applications, because it leads to a locally significant notion of the drift, i.e., the polynomial $\phi(x)$ $=Y(x)-Y_{V}(x), x \in V$. In particular, the estimation of $P(x)$ in a point $x \in V$, or of its coefficients $A_{l}$ is then possible by the technique of the best linear intrinsic estimator (BLIE, see Section 3). There exist $k$-IRF's which cannot be locally stationary. For instance, if an analytic $k$-IRF were locally stationary, it would be stationary on the whole space $\boldsymbol{R}^{\boldsymbol{n}}$.

Theorem 6. Any $k$-IRF with polynomial GC is locally stationary on any bounded open set $V$.

Proof. If the theorem is true in $\boldsymbol{R}^{1}$, it will be true in $\boldsymbol{R}^{n}$ for any $n>0$ by the turning bands method. Then, suppose $n=1$, and examine at first the case $k=0$.

Let $Z$ be a 0 -IRF with the GC $-|h|$ on $\boldsymbol{R}^{1}$, and choose the representation $W(x)=Z\left(\delta_{x}-\frac{1}{2}\left(\delta_{L}+\delta_{0}\right)\right)$, for an arbitrary real $L>0$. The covariance of $W(x)$ satisfies

$$
\langle W(x), W(y)\rangle=\frac{1}{2} L-|x-y|
$$

for $x, y \in[0, L]$, and, in particular, $W(0)+W(L)=0$. Thus, it is possible to define a periodic random function $Y_{0}(x)$ continuous on $R^{1}$ by putting

and

$$
Y_{0}(x)=W(x) \text { if } x \in[0, L]
$$

$$
Y_{0}(x)=(-1)^{k} Y_{0}(x+k L) \text { for } k L \leqq x \leqq(k+1) L, k \text { integer. }
$$

The period of $Y_{0}(x)$ is $2 L$. Let $C$ denote the function with period $2 L$ defined by $C(h)=\frac{1}{2} L-|h|$ for $|h| \leqq L$. For $x=x_{0}+k L, y=y_{0}+k^{\prime} L, x_{0}$, $y_{0} \in[0, L]$, we have

$$
\begin{aligned}
\left\langle Y_{0}(x), Y_{0}(y)\right\rangle & =(-1)^{k+k^{\prime}}\left[\frac{1}{2} L-\left|x_{0}-y_{0}\right|\right] \\
& =(-1)^{k+k^{\prime}}\left[\frac{1}{2} L-\left|x-y-\left(k-k^{\prime}\right) L\right|\right] \\
& =C(|x-y|)
\end{aligned}
$$

by the relationship $C(h+k L)=(-1)^{k} C(h)$.

Thus $Y_{0}(x)$ is a SRF and its covariance is $C(h)$. By $W(x)=Y_{0}(x)$ for $0 \leqq x \leqq L, Z$ is then locally stationary on $[0, L]$. 
Now let $Z$ be a $k$-IRF with polynomial covariance on $\boldsymbol{R}^{1}$, and $W(x)$ the 0 -IRF occurring in its representation (5.3) (Theorem 5) which may be chosen in such a way that $W(0)+W(L)=0$. By the preceding part of the proof, we have $W(x)=Y_{0}(x)$ if $0 \leqq x \leqq L$ for a periodic SRF $Y_{0}(x)$, the covariance of which is $C(h)$. By the Fourier expansion,

$$
C(h)=\sum_{q=0}^{\infty} 4 L \pi^{-2}(2 q+1)^{-2} \cos (2 q+1) \pi(h / L),
$$

there exists a sequence $\left\{C_{l}\right\}, l= \pm 1, \pm 3, \cdots$ of complex orthogonal variables, with $C_{-l}=\bar{C}_{l}$ and $\left\|C_{l}\right\|^{2}=4 L /(\pi l)^{2}$ such that

$$
Y_{0}(x)=\Sigma_{l} C_{l} \exp (-i \pi l x / L) .
$$

For $p$ integer $>0$, the SRF $Y_{p}(x)$ defined by

$$
Y_{p}(x)=\Sigma(i L / \pi l)^{p} C_{l} \exp (-i \pi l x / L)
$$

admits the covariance

$$
C_{p}(h)=4 \sum_{q=0}^{\infty} L^{2 p+1}((2 q+1) \pi)^{-2 p} \cos (2 q+1) \pi(h / L),
$$

and is equal to $\int_{0}^{x}(x-\xi)^{p-1} Y_{0}(\xi) /(p-1) ! d \xi$ up to a random polynomial of degree $\leqq p$. By the formula (5.3), Theorem 5 , the $\operatorname{SRF} \sum b_{p} Y_{p}(x)$ is thus equal to $Y(x)$, up to a random polynomial of degree $\leqq k$ for any $x \in[0, L]$. Thus, $Z$ is locally stationary on $[0, L]$.

Corollary. Let $Z$ be a $k$-IRF with the GC

$$
K(h)=\sum_{0}^{k}(-1)^{p} a_{p}|h|^{2 p+1} /(2 p+1) !
$$

on $\boldsymbol{R}^{1}$, and $C(h)$ the covariance of the SRF with period $2 L$ which coincides on $[0, L]$ with a representation of $Z$. Then,

$$
\begin{aligned}
C(h) & =4 \sum_{q=0}^{\infty} B_{q} \cos (2 q+1) \pi(h / L), \\
B_{q} & =\sum_{p=0}^{k} a_{p} L^{2 p+1} \pi^{-2 p-2}(2 q+1)^{-2 p-2} .
\end{aligned}
$$

The corollary follows easily from Lemma 5 and

$$
\Sigma a_{p} x^{2(k-p)}=\left|\Sigma b_{p}(i x)^{k-p}\right|^{2} .
$$

Note that $C(h)=K(h)$ for $|h| \leqq L$, up to an even polynomial of degree $\leqq 2 k$. 


\section{References}

[1] Cramér, H. AND Leadbetter, M. R. (1969) Stationary and Related Stochastic Processes. Wiley, New York.

[2] Feller, W. (1957) An Introduction to Probability Theory and its Applications. Vol. 1. Wiley, New York.

[3] Guelfand, M. AND Vilenkin, N. Y. (1961) Nekotorye primenenia garmonitsheskovo analisa. Moscow. York.

[4] Guelfand, M. and Vilenkin, N. Y. (1967) Les Distributions. Vol. 4. Dunod, Paris.

[5] Grenander, U. and Rosenblatt, M. (1957) Stationary Time Series. Wiley, New

[6] Guibal, D. (1972) Simulations de schémas intrinsèques. Internal report, Centre de Morphologie Mathématique, Fontainebleau.

[7] HuijBregts, Ch. AND Matheron, G. (1970) Universal Kriging - an optimal method for estimating and contouring in trend surface analysis. CIMM International Symposium, Montreal.

[8] Matheron, G. (1969) Le Krigeage Universel. Fasc. No. 1, Cahiers du Centre de Morphologie Mathématique, Fontainebleau.

[9] Matheron, G. (1971) The theory of regionalized variables, and its applications. Fasc. No. 5, Cahiers du Centre de Morphologie Mathématique, Fontainebleau.

[10] ORfeuil, J. P. (1972) Simulation du Wiener-Lévy et de ses intégrales. Internal report, Centre de Morphologie Mathématique, Fontainebleau.

[11] Yaglom, A. M. (1962) Stationary Random Functions. Prentice Hall, Englewood Cliffs, N. J.

[12] WIENER, N. AND MASANI, P. (1958) The prediction theory for multivariate stochastic processes. I. Acta Math. 98, 111-150; II. Acta Math. 99, 93-137. 